\title{
Respuesta de la relación volumen corriente-tiempo inspiratorio durante un esfuerzo incremental
}

\author{
P.J. Benito ${ }^{\text {a }}$, F.J. Calderón ${ }^{\mathrm{a}}$, A. García-Zapico ${ }^{\mathrm{b}}$, J.C. Legido $^{\mathrm{c}}$ y J.A. Caballero ${ }^{\mathrm{d}}$

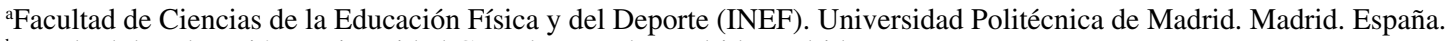 \\ bFacultad de Educación. Universidad Complutense de Madrid. Madrid. España. \\ ${ }^{c}$ Escuela Profesional de Especialistas en Medicina de la Educación Física y del Deporte. Facultad de Medicina. \\ Universidad Complutense de Madrid. Madrid. España. \\ ${ }^{\mathrm{d}}$ Facultad de Ciencias de la Actividad Física y el Deporte. Las Palmas de Gran Canaria. Las Palmas. España.
}

OвJETIVo: La participación del reflejo de Hering-Breuer durante el ejercicio en seres humanos es objeto de discusión. El propósito del presente trabajo ha sido estudiar la respuesta del patrón respiratorio durante un esfuerzo incremental en cicloergómetro para comprobar, de forma indirecta, mediante el análisis de la relación volumen corriente-tiempo inspiratorio $\left(\mathrm{V}_{\mathrm{T}} / t_{\mathrm{I}}\right)$, la participación del reflejo de HeringBreuer en el control de la respiración.

SuJETOS Y MÉTODOs: Han participado en el estudio 9 sujetos activos que han llevado a cabo un protocolo incremental en cicloergómetro hasta alcanzar criterios máximos. Se ha comprobado que la relación $\mathrm{V}_{\mathrm{T}} / t_{\mathrm{I}}$ durante el ejercicio presenta 2 fases con un punto de ruptura, denominado punto de ruptura Hering-Breuer (PHB): fase I, donde el incremento de la ventilación se produce a expensas del aumento del $\mathrm{V}_{\mathrm{T}}$ con ligero descenso del $t_{\mathrm{I}}$, y fase II, durante la cual el incremento ventilatorio se produce tanto por el aumento del $V_{\mathrm{T}}$ como por el descenso del $t_{\mathrm{r}}$.

Resultados: En el estudio, el PHB se alcanzaba a un valor medio ( \pm desviación estándar) del $84,6 \pm 6,3 \%$ respecto al máximo valor de $V_{T}(3.065 \pm 566,8 \mathrm{ml})$ y de un $48 \pm 7,2 \%$ respecto al valor de la capacidad vital forzada medida en la espirometría de reposo. EI PHB se relacionó de forma positiva $(r=0,93)$ con el umbral ventilatorio 2 o umbral de compensación respiratoria.

Conclusiones: Existe relación directa entre el PHB y $\mathrm{V}_{\mathrm{T}} / t_{\mathrm{I}}$. El PHB se relaciona con el umbral ventilatorio 2 , de manera que intervendría en el control del patrón ventilatorio durante el ejercicio. La entrada en funcionamiento del reflejo podría contribuir informando a los centros respiratorios para llevar a cabo el cambio de patrón ventilatorio.

Palabras clave: Patrón respiratorio. Reflejo de Hering-Breuer. Umbral ventilatorio.

\section{Introducción}

El patrón respiratorio se ha estudiado en estado de reposo en humanos ${ }^{1}$, durante ejercicio submáximo ${ }^{2-4}$ y máximo ${ }^{2,3,5}$. Un hallazgo común en todos los estudios

Correspondencia: Dr. P.J. Benito-Peinado.

Facultad de Ciencias de la Educación Física y del Deporte (INEF).

Universidad Politécnica de Madrid.

Martín Fierro, s/n. 28040 Madrid. España.

Correo electrónico: pedroj.benito@upm.es

Recibido: 18-10-2004; aceptado para su publicación: 11-10-2005.
Response of Tidal Volume to Inspiratory Time Ratio During Incremental Exercise

OвJестіve: There is some debate about the participation of the Hering-Breuer reflex during exercise in human beings. This study aimed to investigate breathing pattern response during an incremental exercise test with a cycle ergometer. Participation of the Hering-Breuer reflex in the control of breathing was to be indirectly investigated by analyzing the ratio of tidal volume $\left(V_{\mathrm{T}}\right)$ to inspiratory time $\left(t_{\mathrm{I}}\right)$.

SuBJECTS AND METHODS: The 9 active subjects who participated the study followed an incremental protocol on a cycle ergometer until peak criteria were reached. During exercise, $V_{\mathrm{T}} / t_{\mathrm{i}}$ can be described in 2 phases, separated by activation of the Hering-Breuer reflex (inspiratory off-switch threshold). In phase 1 , ventilation increases because $V_{T}$ increases, resulting in a slight decrease in $t_{\mathrm{I}}$, whereas, in phase 2 , increased ventilation is due to both an increase in $V_{T}$ and a decrease in $t_{\text {. }}$.

RESULTS: The mean (SD) inspiratory off-switch threshold was $84.6 \%(6.3 \%)$ when expressed relative to peak $V_{T}$ (mean, $3065[566.8] \mathrm{mL})$ and $48 \%(7.2 \%)$ relative to the forced vital capacity measured by resting spirometry. The inspiratory offswitch threshold correlated positively $(r=0.93)$ with the second ventilatory threshold, or respiratory compensation point.

CONCLUSIONS: The inspiratory off-switch threshold and $\mathrm{V}_{\mathrm{T}} / t_{\mathrm{i}}$ are directly related to one another. The inspiratory off-switch threshold was related to the second ventilatory threshold, suggesting that the Hering-Breuer reflex participates in control of the breathing pattern during exercise. Activation of the reflex could contribute by signaling the respiratory centers to change the breathing pattern.

Key words: Breathing pattern. Hering-Breuer reflex. Ventilatory threshold. ha sido que la relación volumen corriente-tiempo inspiratorio $\left(\mathrm{V}_{\mathrm{T}} / t_{\mathrm{T}}\right)$ muestra una respuesta similar a la descrita por Clark y Von Euler ${ }^{6}$ cuando se respiraba aire enriquecido con anhídrido carbónico $\left(\mathrm{CO}_{2}\right)$. Estos autores describieron el curso de la relación $\mathrm{V}_{\mathrm{T}} / t_{\mathrm{T}}$ en 2 fases: fase $\mathrm{I}$, de incremento de la ventilación a expensas del $\mathrm{V}_{\mathrm{T}}, \mathrm{y}$ fase II, de aumento de la ventilación principalmente por descenso del $t_{\mathrm{I}}$.

La relación $\mathrm{V}_{\mathrm{T}} / t_{\mathrm{I}}$ puede indicar, indirectamente, la activación del reflejo y muestra 2 fases bien delimitadas por la activación de receptores pulmonares, descritas 
por Von Euler y Clark ${ }^{6,7}$. Este patrón indica, por tanto, el paso de una respiración donde la ventilación aumenta fundamentalmente a expensas del $\mathrm{V}_{\mathrm{T}}$ a una respiración taquipneica. Durante el ejercicio de intensidad creciente, se produce precisamente este cambio de patrón. Hay unanimidad en señalar que a partir de cierta intensidad el $\mathrm{V}_{\mathrm{T}}$ alcanza un valor estable $\mathrm{e}^{8-10} \mathrm{o}$ aumenta ligeramente en ciclistas muy entrenados ${ }^{11}$. A partir de cierta intensidad, aumenta notablemente la participación de la frecuencia respiratoria en el incremento del volumen espirado $\left(\mathrm{V}_{\mathrm{E}}\right)$. Durante el ejercicio, es objeto de controversia la contribución del reflejo de Hering-Breuer en el control de la ventilación, pues en animales con doble denervación (hiliar y de los cuerpos carotídeos) se ha observado cómo se modificaba el patrón respiratorio en los animales sometidos a denervación respecto a los normales ${ }^{12}$. Por otra parte, personas que han recibido un trasplante cardiopulmonar ${ }^{13}$ alcanzan una adecuada ventilación durante el ejercicio mediante un incremento considerable del $\mathrm{V}_{\mathrm{T}} \mathrm{y}$ una reducción de la frecuencia respiratoria, lo que indica la activación de receptores pulmonares en el control respiratorio. Estas 2 evidencias experimentales, en animales y seres humanos, permiten establecer la hipótesis de que, en parte, el incremento de la ventilación durante el ejercicio puede atribuirse a la información procedente de receptores de volumen $\mathrm{u}$ otro tipo que, por vía vagal, informarían a los centros de control de la respiración 7 .

El umbral ventilatorio segundo $\left(\mathrm{VT}_{2}\right)$ es uno de los parámetros más utilizados en la evaluación ergoespirométirca. Se define como la intensidad de la carga en la cual pierde la relación lineal el consumo de oxígeno con la producción de $\mathrm{CO}_{2}{ }^{14-16}$. Creemos que esta variable debería tenerse en cuenta en los estudios de la enfermedad pulmonar obstructiva crónica (EPOC) por su demostrada sensibilidad al entrenamiento ${ }^{11}$.

El objetivo de este estudio ha sido analizar la respuesta del patrón respiratorio para intentar justificar, de forma indirecta, la participación del reflejo de HeringBreuer en la regulación de la respiración y si existe relación con el $\mathrm{VT}_{2}$. La hipótesis inicial consiste en que la relación $\mathrm{V}_{\mathrm{T}} / t_{\mathrm{I}}$ durante un ejercicio dinámico de intensidad creciente presenta una respuesta igual o similar a la observada por primera vez por Clark y Von Euler mediante la inhalación de $\mathrm{CO}_{2}$, que demostraba la activación vagal por vía de receptores de volumen ${ }^{6}$. De confirmarse la relación entre los 2 parámetros del patrón respiratorio, sería importante para poder aplicarla a los enfermos con EPOC.

\section{Sujetos y métodos \\ Sujetos}

Los datos descriptivos de la muestra se encuentran en la tabla I. Participaron 9 sujetos varones ciclistas aficionados, triatletas y estudiantes de educación física que regularmente practican ciclismo. Se informó de la naturaleza del trabajo a todos ellos y firmaron un consentimiento por escrito para participar en el estudio, que se desarrolló según las pautas éticas dictadas en la Declaración de Helsinki de la Asociación Médica Mundial para la investigación con los seres hu$\operatorname{manos}^{17}$.

\section{Medidas de intercambio respiratorio}

Las mediciones de composición y volúmenes del aire espirado se realizaron con un equipo Jaeger Oxycon Pro ${ }^{\circledR}$ (Erich Jaeger, Würzberg, Alemania) . La turbina es bidireccional digital (Triple $\mathrm{V}^{\circledR}$, Erich Jaeger, Würzberg, Alemania), de bajo espacio muerto y resistencia, y cumple la normativa de la American Thoracic Society y la European Community for Steel and Coal ${ }^{18}$. Todos los parámetros de intercambio de gases se midieron respiración a respiración y se promediaron cada $15 \mathrm{~s}$. La frecuencia cardíaca se determinó con un equipo de electrocardiografía, modelo Hellige Cardiotest EK 53 (Hellige, Freiburg, Alemania) de 3 canales.

\section{Protocolo de estudio}

A la llegada al laboratorio, se realizó a cada sujeto un examen médico, consistente en la historia clínica y deportiva, electrocardiograma basal y espirometría. Las pruebas se realizaron sobre un cicloergómetro Jaeger ER800 ${ }^{\circledR}$ (Erich Jaeger, Alemania) de resistencia variable y freno electromagnético con un rango de 25 a $1.000 \mathrm{~W}$ e incrementos mínimos de 1 W/s. El protocolo de esfuerzo usado consistió en 1 min totalmente en reposo, 3 min de calentamiento a $50 \mathrm{~W}$ y prueba progresiva con incrementos de $5 \mathrm{~W}$ cada $12 \mathrm{~s}$. Finalizado el esfuerzo, se realizaban 2 min de recuperación activa a $50 \mathrm{~W}(70$ rpm) y 3 min en reposo completo en la bicicleta. La cadencia de pedaleo se estableció entre 70 y $90 \mathrm{rpm}$. Todas las pruebas se llevaron a cabo en condiciones atmosféricas similares (21$24{ }^{\circ} \mathrm{C}$, un $45-55 \%$ de humedad relativa y presión atmosférica entre 700 y $715 \mathrm{mmHg}$ ). Los valores se expresaron en condiciones STPD (standard temperature and pressure dry).

\section{Análisis de la transición aeróbica-anaeróbica}

Los datos de intercambio de gases se analizaron con una técnica de identificación visual propuesta por Wassermann et $\mathrm{al}^{19}$ en 1973. En este método se relacionan $\mathrm{V}_{\mathrm{E}} /$ producción de $\mathrm{CO}_{2}, \mathrm{~V}_{\mathrm{E}}$ /consumo de oxígeno, la presión espiratoria total de $\mathrm{CO}_{2}$ y la presión espiratoria total de oxígeno respecto a la carga, para determinar la transición aeróbica-anaeróbica, usando los criterios habituales. Se ha demostrado que la combinación de diversos métodos es más fiable en la determinación de los umbrales ventilatorios ${ }^{15}$.

Para cada sujeto se representaron los valores correspondientes al $\mathrm{V}_{\mathrm{T}}$ respecto al $t_{\mathrm{I}}$ de la prueba de esfuerzo realizado en función de sus datos individuales, al objeto de determinar de manera visual las 2 fases en el patrón respiratorio adoptado mediante la inhalación de $\mathrm{CO}_{2}$ propuesto por Clark y Von Euler6: a) fase I, donde los incrementos de la ventilación se producen a expensas del aumento del $\mathrm{V}_{\mathrm{T}}$ con un ligero descenso del $t_{\mathrm{I}}, \mathrm{y} b$ ) fase II, en la que el incremento ventilatorio se produce tanto por el aumento del $\mathrm{V}_{\mathrm{T}}$ como por el descenso del $t_{\mathrm{I}}$, siendo la relación inversa. Esta variación en la respuesta ventilatoria se alcanza cuando el $\mathrm{V}_{\mathrm{T}}$ es unas 2 veces el valor de reposo.

En el patrón de Clark y Von Euler el paso de fase se produce bruscamente; es el denominado punto de ruptura HeringBreuer (PHB). Utilizando el método de inspección visual se determinó el punto donde la relación $\mathrm{V}_{\mathrm{T}} / t_{\mathrm{I}}$ se desplaza hacia la izquierda en un diagrama de coordenadas, es decir, el punto donde el $t_{\mathrm{I}}$ disminuye de manera significativa con un pequeño incremento del $\mathrm{V}_{\mathrm{T}}$. Dos observadores ciegos e independientes determinaron el $\mathrm{VT}_{2}$ y el PHB en momentos diferentes sin conocer la identidad de cada sujeto.

\section{Análisis estadístico}

Se calcularon las medias ( \pm desviación estándar) de todas las variables. Tras realizar el estudio de normalidad se aplicó la prueba de la t de Student para muestras relacionadas apare- 


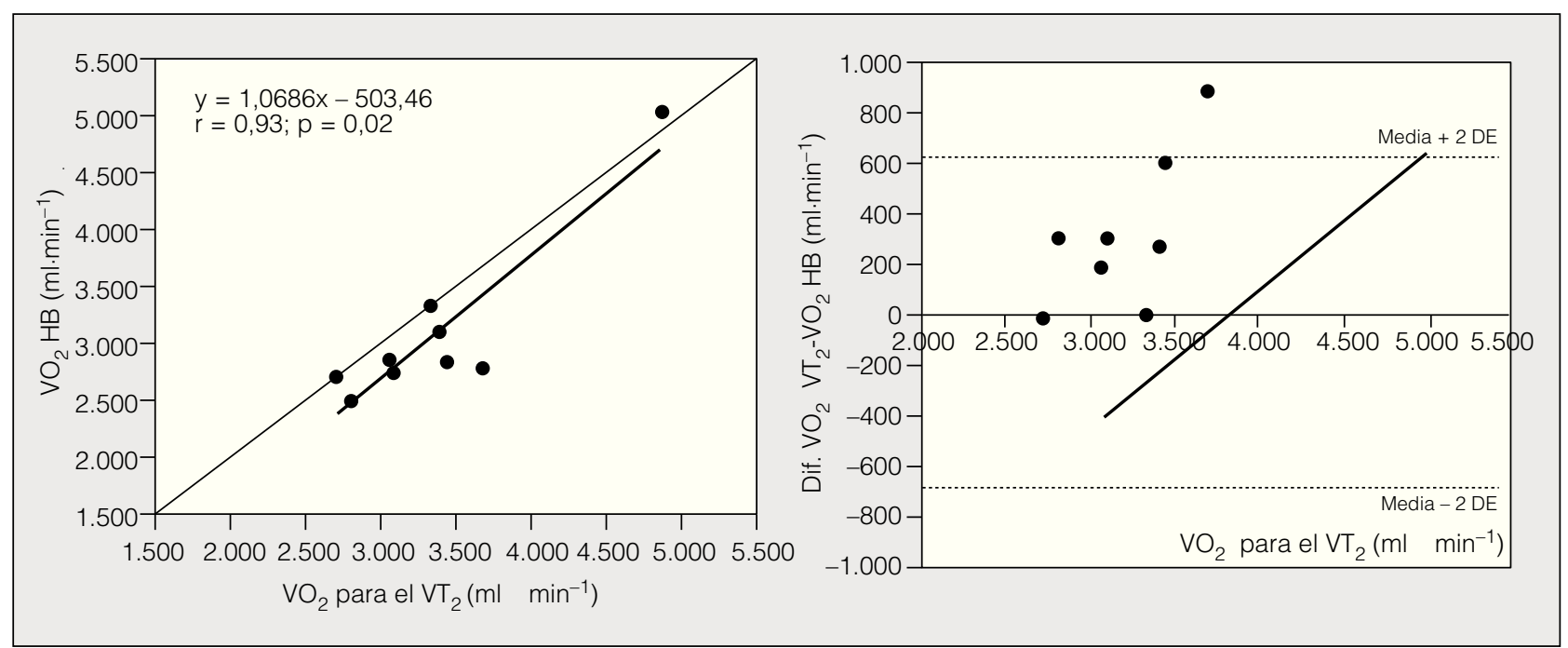

Fig. 1. En la parte izquierda se muestra la relación entre el consumo de oxígeno $\left(\mathrm{VO}_{2}\right)$ en que se produce el punto de Hering-Breuer (HB) y el VO en que se produce el umbral ventilatorio segundo $\left(\mathrm{VT}_{2}\right)$. En la parte derecha se muestra el procedimiento de Bland y Altman (1986) para el análisis de la fiabilidad en el procedimiento de identificación del $\mathrm{VT}_{2}$.

adas. También se realizó un estudio de correlación de Pearson para establecer si existían relaciones positivas entre la transición aeróbica-anaeróbica y el PHB. Se utilizó el procedimiento de Bland y Altam $^{20}$ para analizar la fiabilidad del análisis del $\mathrm{VT}_{2}$ a través de las gráficas de $\mathrm{V}_{\mathrm{T}} / t_{\mathrm{I}}$. El nivel de significación fue de $\mathrm{p}<0,05$. Para el análisis estadístico se utilizó el programa SPSS versión 11.5 (SPSS Worldwide Headquarters, Chicago, EE.UU.).

TABLA I

Características de los sujetos incluidos en el estudio

\begin{tabular}{|l|c|}
\hline & Media $\pm \mathbf{D E}$ \\
\hline Edad (años) & $24,1 \pm 3$ \\
Peso $(\mathrm{kg})$ & $70,5 \pm 6,5$ \\
Estatura $(\mathrm{cm})$ & $176 \pm 6,6$ \\
$\mathrm{FVC}(\mathrm{l})$ & $5,4 \pm 0,9$ \\
$\mathrm{FEV}$ & $(\mathrm{l})$ \\
$\mathrm{VO}_{2}\left(\mathrm{ml} \cdot \mathrm{kg} \cdot \mathrm{min}^{-1}\right)$ & $4,6 \pm 0,6$ \\
Carga máxima $(\mathrm{W})$ & $57,1 \pm 12,1$ \\
\hline
\end{tabular}

FVC: capacidad vital forzada; $\mathrm{FEV}_{1}$ : volumen espiratorio forzado en el primer segundo; $\mathrm{VO}_{2}$ : consumo de oxígeno relativo al peso corporal; carga máxima: promedio de carga máxima desarrollada en las pruebas; DE: desviación estandar.

TABLA II

Resultados del análisis de la relación entre el volumen corriente $\left(V_{t}\right)$ y el tiempo inspiratorio en la prueba incremental máxima y análisis de comparación de medias

\begin{tabular}{|c|c|c|c|c|}
\hline & $\underset{\left(\mathbf{m l} \times \mathbf{m i n}^{-1}\right)}{\mathbf{V O}_{2} \mathbf{H B}}$ & $\underset{\left(\mathbf{m l} \times \mathbf{m i n}^{-1}\right)}{\mathbf{V O}_{2} \mathbf{V T}_{2}}$ & $\begin{array}{c}\mathbf{V}_{\mathbf{t}} \mathbf{H B} \\
(\mathbf{m l})\end{array}$ & $\mathbf{V}_{\mathbf{t}}$ máximo \\
\hline Media & 3.103 & $3.375^{*}$ & 2.576 & 3.065 \\
\hline \multirow[t]{2}{*}{$\mathrm{DE}$} & 751 & 637 & 441 & 566 \\
\hline & $\begin{array}{c}\mathrm{V}_{\mathrm{t}} \mathbf{H B} / \\
\mathrm{V}_{\mathrm{t}} \text { máximo }\end{array}$ & $\begin{array}{l}\mathrm{V}_{\mathrm{t}} \mathrm{HB} / \\
\mathrm{FVC}\end{array}$ & $\begin{array}{l}V_{t} \text { máximo/ } \\
\text { FVC }\end{array}$ & $\begin{array}{l}\mathrm{V}_{\mathrm{t}} \text { máximo/ } \\
\text { FEV }_{1}\end{array}$ \\
\hline Media & $84,6 \%$ & $48,0 \%$ & $56,8 \%$ & $66,1 \%$ \\
\hline DE & $6,3 \%$ & $7,2 \%$ & $7,6 \%$ & $8,1 \%$ \\
\hline
\end{tabular}

$\mathrm{VO}_{2} \mathrm{HB}$ : consumo de oxígeno en el que se produce el punto de Hering-Breuer; $\mathrm{VO}_{2} \mathrm{VT}$. consumo de oxígeno en el que se produce el umbral ventilatorio segun$\mathrm{VO}_{2} \mathrm{VT}_{2}$ : consumo de oxígeno en el que se produce el umbral ventilatorio segun-
do; $\mathrm{V}_{\mathrm{t}} \mathrm{HB}$ : $\mathrm{V}_{\mathrm{t}}$ en el punto de Hering-Breuer; FVC: capacidad vital forzada; $F E V_{1}$ : volumen espiratorio forzado en el primer segundo; DE: desviación estándar. *Diferencias significativas $\mathrm{VO}_{2} \mathrm{HB}$ frente a $\mathrm{VO}_{2} \mathrm{VT}_{2}: \mathrm{t}(8)=2,559 ; \mathrm{p}=0,034$

\section{Resultados}

En la tabla I se muestran los datos de antropometría, espirometría de reposo y consumo máximo de oxígeno de la población estudiada. Los valores de consumo máximo de oxígeno son superiores $\left(57,1 \pm 12,4 \mathrm{ml} \cdot \mathrm{kg} \cdot \mathrm{min}^{-1}\right)$ a los correspondientes a la población sedentaria, alcanzando una carga media de $336 \mathrm{~W}$. En la tabla II se recogen los valores correspondientes al momento en que se producía el cambio de fase del patrón respiratorio. En cada una de las pruebas realizadas se distinguieron las 2 fases señaladas anteriormente en el apartado "Sujetos y métodos".

El PHB se producía cuando el $t_{\mathrm{I}}$ era de $0,96 \pm 0,11 \mathrm{~s}$, correspondiente a un $\mathrm{V}_{\mathrm{T}}$ de $2.576 \pm 441 \mathrm{ml}$. Este volumen supone un porcentaje medio del $84,6 \pm 6,3 \%$ en relación con el máximo valor de $\mathrm{V}_{\mathrm{T}}(3.065 \pm 566 \mathrm{ml})$ alcanzado durante la prueba máxima, y de un $48 \pm 7,2 \%$ respecto al valor de capacidad vital forzada medida en la espirometría de reposo $(5.416 \pm 823 \mathrm{ml})$. El $\mathrm{V}_{\mathrm{T}}$ máximo durante la prueba fue un $56,8 \pm 7,6 \%$ respecto a la capacidad vital forzada.

El PHB se relacionó de forma positiva $(\mathrm{r}=0,93 ; \mathrm{p}=$ 0,02 ) con el $\mathrm{VT}_{2}$ (fig. 1). La prueba de Bland y Altman demuestra que tan sólo en un sujeto existen diferencias entre el valor de ambas medidas. En la tabla II se muestra el consumo de oxígeno medio en el que se produce el PHB, que precede en aproximadamente $300 \mathrm{ml}$ al consumo de oxígeno medio donde se produce el $\mathrm{VT}_{2}$.

\section{Discusión}

En este estudio se ha demostrado que el comportamiento de la relación $\mathrm{V}_{\mathrm{T}} / t_{\mathrm{I}}$ durante un esfuerzo de intensidad creciente es similar al observado por Clark y Von Euler en animales sometidos a inhalación de $\mathrm{CO}_{2}$. La respuesta de la relación muestra 2 fases bien diferenciadas (fig. 2). En la fase I, el incremento del $V_{E}$ se produce por un aumento, principalmente, del $\mathrm{V}_{\mathrm{T}} \mathrm{y}$ una 


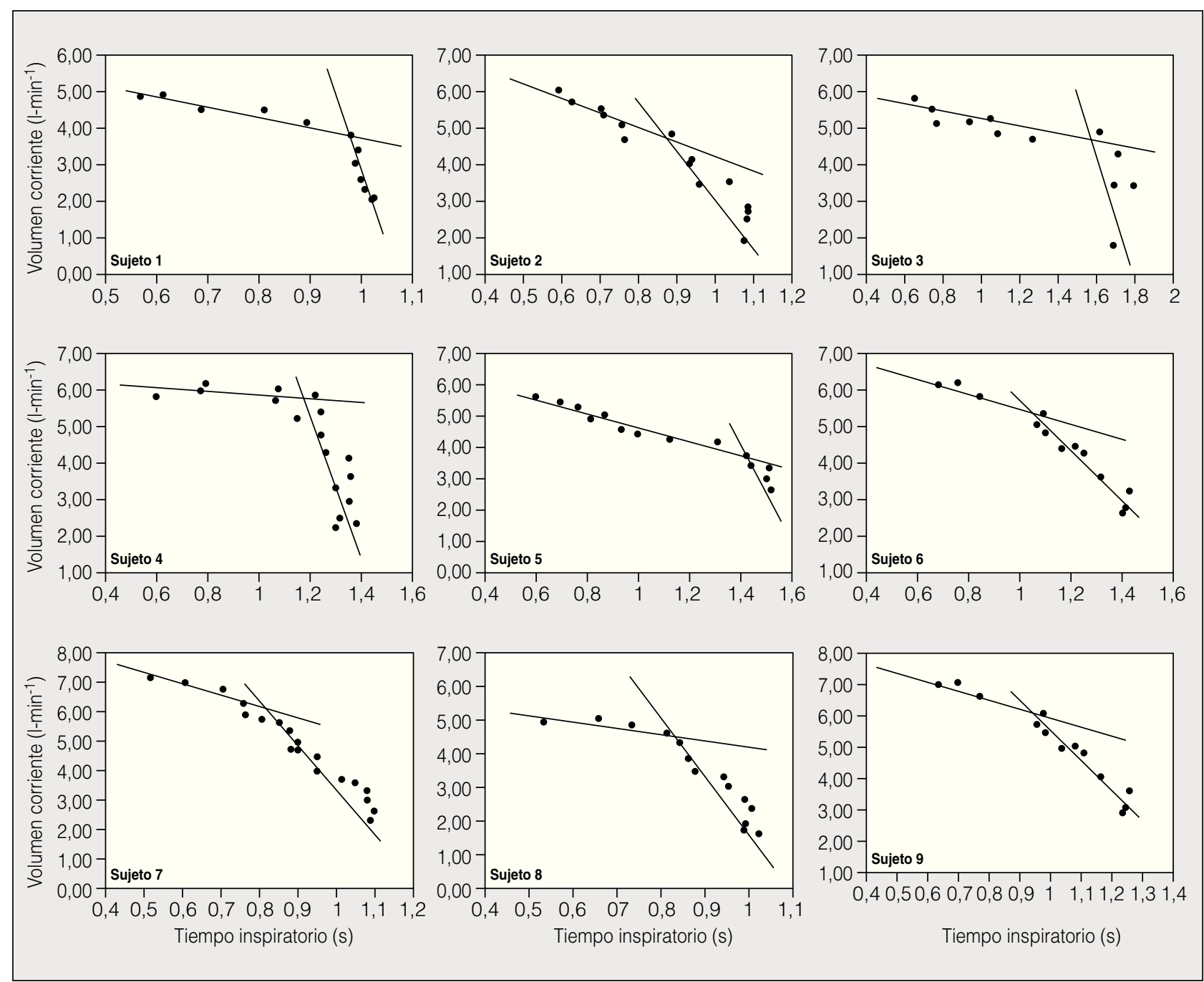

Fig. 2. Gráficas de la relación entre volumen corriente y tiempo inspiratorio de los sujetos que participaron en este estudio. Las líneas punteadas corresponden a las ecuaciones de regresión con las que se determinó el punto de incidencia del reflejo de Hering-Breuer. Las escalas se ajustaron para observar más correctamente el fenómeno de manera individual.

reducción del $t_{\mathrm{I}}$. Por el contrario, en la fase II la hiperventilación es el resultado de una mayor reducción del $t_{\mathrm{I}}$ con un ligero incremento del $\mathrm{V}_{\mathrm{T}}$. El momento en que se cambia de fase, se denomina PHB, y en el estudio realizado se corresponde con el $\mathrm{VT}_{2}$. Hay unanimidad en señalar que a partir de cierta intensidad el $\mathrm{V}_{\mathrm{T}}$ alcanza un valor estable $e^{9,10,21}$ o aumenta ligeramente ${ }^{11}$ en función del grado de entrenamiento. Algunos autores han demostrado que el entrenamiento de alta intensidad puede aumentar el $\mathrm{V}_{\mathrm{T}}$ en enfermos con EPOC ${ }^{22}$. Sin embargo, otros investigadores no han observado la modificación del patrón respiratorio en esfuerzo ${ }^{23}$ o bajo anestesia ${ }^{10}$, pero sí en personas con trasplante cardiopulmonar ${ }^{13,24}$. Las divergencias entre los resultados obtenidos por Kay et $\mathrm{al}^{23}$ y los del presente estudio podrían deberse a que en su caso el protocolo de esfuerzo era de carga limitada, de 50 a $200 \mathrm{~W}$, mientras que el protocolo aplicado por nosotros ha sido incremental máximo.

Por otra parte, el estudio realizado no permite establecer de forma concluyente la intervención de reflejos vagales en el control ventilatorio durante el ejercicio, debido a que, por tratarse de un estudio con seres humanos, no han podido controlarse muchas variables que podrían explicar el comportamiento de la relación $\mathrm{V}_{\mathrm{T}} / t_{\mathrm{I}}$. Los mecanismos fisiológicos propuestos de regulación de la respiración en ejercicio no explican completamente la respuesta del $\mathrm{V}_{\mathrm{E}} \mathrm{y}$ se pueden agrupar en: prealimentación (feedforward), retroalimentación (feedback) y potenciación a corto plazo (short term potentiation $)^{25}$. La retroalimentación puede proceder de las propias estructuras del sistema nervioso central (retroalimentación central) o de receptores localizados en el aparato respiratorio (parénquima y tórax), en la musculatura de la locomoción y en el sistema cardiovascular (quimiorreceptores periféricos), es decir, retroalimentación periférica. Por tanto, no hemos podido controlar la información de: a) las terminaciones III de la musculatura implicada; $b$ ) los quimiorreceptores periféricos, y c) los receptores sensibles a determinadas moléculas e iones (potasio, protones). 
A pesar de las limitaciones señaladas, los resultados del estudio demuestran que la relación $\mathrm{V}_{\mathrm{T}} / t_{\mathrm{I}}$ en esfuerzo de intensidad creciente sigue un patrón bifásico con un punto de ruptura a un valor de $\mathrm{V}_{\mathrm{T}}$ del $84,6 \pm 6,3 \%$ respecto al máximo alcanzado. Consideramos que una posible explicación sería la activación del reflejo de Hering Breuer. La intervención de este mecanismo reflejo en el control respiratorio se ha demostrado en animales de experimentación ${ }^{26}$. Las razones fisiológicas en que apoyamos la intervención del reflejo de Hering-Breuer para explicar los resultados tienen su base en que la relación inversa entre el $\mathrm{V}_{\mathrm{T}}$ y el $t_{\mathrm{I}}$ se ve alterada en diversas condiciones experimentales (vagotonía, denervación hiliar, hipercapnia, bloqueo de receptores beta, presión generada en la boca), tanto en animales como en seres humanos.

La vagotonía determina el incremento del $\mathrm{V}_{\mathrm{E}}$ a expensas del $\mathrm{V}_{\mathrm{T}}$ con un descenso mínimo del $t_{\mathrm{I}}$, de manera que la relación $\mathrm{V}_{\mathrm{T}} / t_{\mathrm{I}}$ estaría en zona $\mathrm{I}^{13,27}$. En un interesante estudio ${ }^{12}$ encaminado a demostrar los efectos sobre la respiración de la doble denervación - hiliar y de los quimiorreceptores carotídeos-, se comprobó que las aferencias pulmonares vía nervios del hilio influían en el patrón respiratorio de los ponis en reposo y esfuerzo. Además, el efecto se relacionaba con la atenuación de la retroalimentación del volumen pulmonar.

La hipercapnia, tanto en animales ${ }^{28}$ como en seres humanos $^{9}$, demuestra la participación vagal en el control de la respiración durante el ejercicio. Joyner et al $^{29}$ demostraron que la administración de bloqueadores beta de receptores 1 y 2 determinaba reducciones más importantes de $\mathrm{V}_{\mathrm{T}}$ que la de fármacos bloqueadores beta específicos de los receptores 1 .

Estudios realizados mediante la técnica más sencilla, esto es, la presión generada en la boca a $0,1 \mathrm{~s}^{5,30,31}$ para valorar la relación $\mathrm{V}_{\mathrm{T}} / t_{\mathrm{I}}$, demuestran que en esfuerzos ligeros los incrementos de la ventilación se producen por aumentos de $\mathrm{V}_{\mathrm{T}}$.

Los resultados de nuestro estudio permiten conjeturar, con la base científica de los resultados experimentales comentados, la importancia de la información procedente de receptores localizados en las vías respiratorias y parénquima pulmonar. La modificación del patrón respiratorio a partir del PHB podría deberse a la retroalimentación originada en receptores de volumen u otro tipo de receptores que podrían ser, en parte, responsables del descenso del $t_{\mathrm{I}}$ cuando se incrementa el $\mathrm{V}_{\mathrm{T}}$. El papel del reflejo de Hering-Breuer en la regulación de la respiración en reposo ha suscitado gran controversia ${ }^{26}$, debido a que los receptores no sólo descargan con los cambios de volumen, sino también con variaciones de la concentración de determinadas moléculas. Por consiguiente, otras posibilidades fisiológicas de los resultados obtenidos en relación con el PHB pueden ser las siguientes: variaciones en la concentración de $\mathrm{CO}_{2}$ y aumento de la concentración de determinados parámetros.

Se ha demostrado que cambios ligeros de la presión parcial de $\mathrm{CO}_{2}$ estimulan los receptores implicados en el reflejo de Hering-Breuer ${ }^{32}$, los cuales, por vía vagal, podrían desencadenar una mayor activación de los centros bulbares de la respiración. En nuestro estudio, la relación del PHB con el $\mathrm{VT}_{2}$, a partir del cual se eleva la presión espiratoria total de $\mathrm{CO}_{2}$, indica que han podido producirse variaciones en la presión parcial del $\mathrm{CO}_{2}$ que justificarían la estimulación de receptores pulmonares sensibles a este gas.

A intensidades elevadas, aumenta la concentración de protones en plasma (acidosis metabólica) y se produce una variación en la concentración de potasio, que puede alcanzar valores tan elevados como $7 \mathrm{mEq} / \mathrm{l}$, lo cual podría explicar en parte la hiperventilación en esfuerzo, bien por estimulación de los quimiorreceptores periféricos o centrales ${ }^{33,34}$. En nuestro estudio no hemos obtenido parámetros del estado ácido-base, de manera que no podemos saber si se ha producido un aumento de la concentración de potasio y de protones en sangre que justificara el cambio en el patrón respiratorio. No obstante, la presión espiratoria total de $\mathrm{CO}_{2}$ cambia a partir del punto de ruptura, de manera que una ligera oscilación de este parámetro podría explicar la estimulación de receptores de volumen u de otro tipo al $\mathrm{CO}_{2}{ }^{35}$.

También se ha demostrado que puede utilizarse el procedimiento de análisis del $\mathrm{VT}_{2}$ a través de la relación $\mathrm{V}_{\mathrm{T}} / t_{\mathrm{I}}$, aunque con cierta cautela, ya que el PHB precede al primero en aproximadamente $300 \mathrm{ml}$, muy probablemente como proceso de anticipación de la ventilación. Nuestros resultados de la relación entre el $\mathrm{V}_{\mathrm{T}}$ y la capacidad vital forzada están de acuerdo con los presentados por otros autores ${ }^{36}$. Estos demostraron que existen diferencias significativas entre personas con y sin EPOC. Esta variable ha demostrado ser un buen indicador de la mejora de la ventilación con el entrenamiento ${ }^{22}$. Por tanto, el PHB puede ser un procedimiento para ver la evolución de los pacientes con EPOC.

En conclusión, la relación $\mathrm{V}_{\mathrm{T}} / t_{\mathrm{I}}$ durante un ejercicio de intensidad creciente muestra un comportamiento en 2 fases: en la primera se produce un aumento de la ventilación a expensas del $\mathrm{V}_{\mathrm{T}} \mathrm{y}$, en menor medida, un incremento de la frecuencia respiratoria (reducción del $t_{\mathrm{I}}$ ); en la segunda, aumenta la frecuencia respiratoria, lo que condiciona el incremento del $\mathrm{V}_{\mathrm{T}}$. El paso de fase se muestra por un punto de ruptura, denominado PHB, que se relaciona con el $\mathrm{VT}_{2}$. Siendo conscientes de las limitaciones del estudio, proponemos que el PHB puede deberse a la estimulación de receptores de volumen, de manera que determinado grado de distensión pulmonar podría informar a los centros de regulación al objeto de incrementar la ventilación. Este procedimiento podría aplicarse a los enfermos con EPOC.

\section{BIBLIOGRAFÍA}

1. Von Euler C. On the central pattern generator for the basic breathing rhythmicity. J Appl Physiol. 1983;55:1647-59.

2. Askanazi J, Milic-Emili J, Broell JR, Hyman AI, Kinney JM. Influence of exercise and $\mathrm{CO}_{2}$ on breathing pattern of normal man. J Appl Physiol. 1979; 47:192-6.

3. Clark JM, Hagerman FC, Gelfand R. Breathing patterns during submaximal and maximal exercise in elite oarsmen. J Appl Physiol. 1983;55:440-6.

4. Ellingsen I, Sydnes G, Hauge A, Nicolaysen G. Effects of exercise and $\mathrm{CO}_{2}$ inhalation on the breathing pattern in man. Acta Physiol Scand. 1988;134:161-73.

5. Lind F, Hesser CM. Breathing pattern and lung volumes during exercise. Acta Physiol Scand. 1984;120:123-9. 


\section{BENITO PJ ET AL. RESPUESTA DE LA RELACIÓN VOLUMEN CORRIENTE-TIEMPO INSPIRATORIO}

DURANTE UN ESFUERZO INCREMENTAL

6. Clark FJ, Von Euler C. On the regulation of depth and rate of breathing. J Physiol. 1972;222:267-95.

7. Dempsey JA, Adams L, Ainsworth D, Fregosi RF, Gallagher CG, Guz A, et al. Airway, lung and respiratory muscle function during exercise. En: Cheniack NS, Widdicombe JG, editors. Handbook of physiology exercise: regulation and integration of multiple systems. Bethesda: American Physiological Society; 1986. p. 69-91.

8. Gallagher CG, Brown E, Younes M. Breathing pattern during maximal exercise and during submaximal exercise with hypercapnia. J Appl Physiol. 1987;63:238-44.

9. Martin BJ, Weil JV. $\mathrm{CO}_{2}$ and exercise tidal volume. J Appl Physiol. 1979;46:322-5.

10. Polacheck J, Strong R, Arens J, Davies C, Metcalf I, Younes M. Phasic vagal influence on inspiratory motor output in anesthetized human subjects. J Appl Physiol. 1980;49:609-19.

11. Lucía A, Carvajal A, Calderón FJ, Alfonso A, Chicharro JL. Breathing pattern in highly competitive cyclists during incremental exercise. Eur J Appl Physiol Occup Physiol. 1999;79:512-21.

12. Flynn C, Forster HV, Pan LG, Bisgard GE. Role of hilar nerve afferents in hyperpnea of exercise. J Appl Physiol. 1985;59:798806.

13. Sciurba FC, Owens GR, Sanders MH, Griffith BP, Hardesty RL, Paradis IL, et al. Evidence of an altered pattern of breathing during exercise in recipients of heart-lung transplants. N Engl J Med. 1988;319:1186-92.

14. Beaver WL, Wasserman K, Whipp BJ. A new method for detecting anaerobic threshold by gas exchange. J Appl Physiol. 1986; 60:2020-7.

15. Gaskill SE, Ruby BC, Walker AJ, Sánchez OA, Serfass RC, Leon AS. Validity and reliability of combining three methods to determine ventilatory threshold. Med Sci Sports Exerc. 2001;33:1841-8.

16. Wasserman K, Hansen JE, Sue D Y, Whipp BJ, Casaburi R. Principles of exercise testing and interpretation. $2^{\text {nd }}$ ed. Philadelphia: Lea \& Febiger; 1994.

17. WMA. Declaración de Helsinki para la investigación con seres humanos. 2000. Disponible en: http://www.wma.net/s/policy/b3.htm

18. Quanjer GJ, Tammeling JE, Cotes LM, Fabbri H, Matthys OF, Pedersen $\mathrm{R}$ et al. Lung volume and forced ventilatory flows. Report working party standarization of lung funtion tests, European Community for Steel and Coal. Eur Respir J Suppl. 1993;16:5-40.

19. Wasserman K, Whipp BJ, Koyl SN, Beaver WL. Anaerobic threshold and respiratory gas exchange during exercise. J Appl Physiol. 1973;35:236-43.

20. Bland JM, Altman DJ. Regression analysis. Lancet. 1986;19:908-9.

21. Gallagher CG. Exercise and chronic obstructive pulmonary disease. Med Clin North Am. 1990;74:619-41.

22. Ruiz de Ona Lacasta JM, García de Pedro J, Puente Maestu L, Llorente Iñigo D, Celdrán Gil J, Cubillo Marcos JM. Effects of muscle training on breathing pattern in patients with severe chronic obstructive pulmonary disease. Arch Bronconeumol. 2004;40:20-3.
23. Kay JD, Petersen ES, Vejby-Christensen H. Breath-by-breath pattern in man during steady-state bicycle exercise. J Physiol. 1975; 244:52P-3P.

24. Sciurba FC, Owens GR, Sanders MH, Costantino JP, Paradis IL, Griffith BP. The effect of obliterative bronchiolitis on breathing pattern during exercise in recipients of heart-lung transplants. Am Rev Respir Dis. 1991;144:131-5.

25. Eldridge FL, Waldrop TG. Neural control of breathing during exercise. En: Whipp BJ, Wasserman K, editors. Exercise: pulmonary physiology and pathophysiology. New York: Marcel Dekker, Inc.; 1991. p. 309-70.

26. Coleridge HM, Coleridge JCG. Reflexes evoked from tracheobronchial tree and lungs. En: Fishman AP, editor. Handbook of physiology. Bethesda: American Physiological Society; 1986. p. 407-13.

27. Clifford PS, Litzow JT, Von Colditz JH, Coon RL. Effect of chronic pulmonary denervation on ventilatory responses to exercise. J Appl Physiol. 1986;61:603-10.

28. Ainsworth DM, Smith CA, Johnson BD, Eicker SW, Henderson KS, Dempsey JA. Vagal modulation of respiratory muscle activity in awake dogs during exercise and hypercapnia. J Appl Physiol. 1992;72:1362-7.

29. Joyner MJ, Jilka SM, Taylor JA, Kalis JK, Nittolo J, Hicks RW, et al. Beta-blockade reduces tidal volume during heavy exercise in trained and untrained men. J Appl Physiol. 1987;62:1819-25.

30. Milic-Emili J, Zin WA. Relationship between neuromuscular respiratory drive and ventilatory output. En: Fishman AP, Macklem PT, Mead J, Geiger SR, editors. Handbook of physiology. The respiratory system. Bethesda: American Physiological Society; 1986. p. 631-46.

31. Whitelaw WA, Derenne JP, Milic-Emili J. Occlusion pressure as a measure of respiratory center output in conscious man. Respir Physiol. 1975;23:181-99.

32. Matsuoka T, Mortola JP. Effects of hypoxia and hypercapnia on the Hering-Breuer reflex of the conscious newborn rat. J Appl Physiol. 1995;78:5-11.

33. McLoughlin P, Popham P, Linton RA, Bruce RC, Band DM. Exercise-induced changes in plasma potassium and the ventilatory threshold in man. J Physiol. 1994;479:139-47.

34. Paterson DJ. Potassium and breathing in exercise. Sports Med. 1997;23:149-63

35. Kaufman MP, Forster HV. Reflexes controlling circulatory. Ventilatory and airway responses to exercise. En: Rowell LB, Mitchell $\mathrm{JH}$, editors. Handbook of physiology. Exercise: regulation and integration of multiple systems. New York: Oxford University Press; 1996. p. 333-80.

36. González-García M, Barrero M, Maldonado D. Exercise limitation in patients with chronic obstructive pulmonary disease at the altitude of Bogota $(2,640 \mathrm{~m})$. Breathing pattern and arterial gases at rest and peak exercise. Arch Bronconeumol. 2004;40:54-61. 